\section{The impact of elderly social care in the United Kingdom: migrants' perspective}

\author{
Osinachi Akanwa Ekeagwu, \\ Stanley Idika Ukpai
}

Anglia Ruskin University, Cambridge, UK

\begin{abstract}
Due to the growing elderly population in the United Kingdom, there is increasing demand for formal carers. Research suggests that elderly care may be associated with adverse impacts, however not much is known about migrants who constitute a considerable proportion of formal carers. Given that migrants already face peculiar challenges related to cultural assimilation and social integration, it becomes necessary to investigate the impact of a burdensome yet well sought job among them. This study therefore aimed to investigate the impact (burden and benefit) of elderly social care among migrant carers.
\end{abstract}

A descriptive cross-sectional survey design was used and data obtained through snowball sampling using web-based questionnaires.

Majority of participants $(51.6 \%)$ confirmed that the choice of the job was financially motivated, even though most (63.3\%) experienced stress. Apparently, the perceived stress was related to frustrations stemming from their skills not being fully utilised, but unrelated to gender, prior experience and cultural background. Nevertheless, $66.7 \%$ remained happy to recommend their job to others.

The inherent caregiver burden should be recognised and addressed by all parties involved including the carers, employers and policy makers. Although the avoidance of caregiver burden entirely may be impossible, there should be room for decreasing this impact and providing respite for those already affected.

\section{Introduction}

In parts of Europe including the United Kingdom, elderly care is largely reliant on the supply of migrant labour. ${ }^{1}$ The UK government worker registration reveals that social care work was the 8th most popular job amongst migrants living in England in 2008 and that $20 \%$ of care workers were born out- side the United Kingdom. ${ }^{2}$ Clausen et al. ${ }^{3}$ explain that some migrants consider care work as an opportunity for first work experience and a means to prepare themselves for the labour market. Apparently, the uptake of such job by migrants has been favoured by increased vacancies created by a shortage of locals in care. ${ }^{4}$ Although it may be argued that the growing ageing population is a contributor, Mcgregor ${ }^{5}$ admits that the social care industry in the past decade has undergone certain changes leading to the creation of challenging employment conditions causing those with better employment prospects to quit. In other words, locals with better employment prospects are apt to quit care job roles for migrant counterparts who have relatively unfavourable employment prospects. ${ }^{6}$ The nature of such challenging environment created by the social care industry is unclear, but agreeably can be deduced from the experiences of present serving carers.

Research abounds regarding the experiences of informal carers particularly relating to caregiver burden. Chiatti et al. ${ }^{7}$ for instance, explored the impact of migrant care workers on the burden of members of Italian families caring for a disabled older person, while some have associated variables with care burdens such as social situation, frequency, and intensity of care ${ }^{8}$ individual and environmental factors ${ }^{9}$ in Spain and Denmark respectively. The stated studies though pertain to elderly individuals, neither of them is based in the United Kingdom, or at least they failed to state it. Others ${ }^{10,11}$ have merely suggested that elderly social care may result in adverse physical and psychological effects, but have not explored these in any migrant population.

The International Organisation for Migration (IOM) recognises a migrant as one who is moving or has moved over international borders, away from their habitual place of residence. ${ }^{12}$ Irrespective of their intent for moving, migrants face challenges related to pre-migration, migration and postmigration stressors. ${ }^{13}$ Although the premigration and migration stressors are arguably inherent in the short-term, postmigration stressors such as cultural assimilation, economic and social integration can linger constituting a considerable burden. In addition to such burden, undertaking a stressful job role such as elderly care could increase their risk of health and psychological problems. Hence the need to investigate the impact of the job on them as it appears to be well sought by the group.

The present study investigates the impact of working in elderly care on migrants. International students working in elderly social care are used to mirror the migrant population. These students are
Correspondence: Osinachi Akanwa Ekeagwu, Anglia Ruskin University, Cambridge, United Kingdom.

E-mail: osinachi.ekeagwu@pgr.anglia.ac.uk

Key words: Caregiver; elderly social care; migrant.

Received for publication: 27 January 2018. Revision received: 5 March 2018.

Accepted for publication: 5 March 2018

This work is licensed under a Creative Commons Attribution-NonCommercial 4.0 International License (CC BY-NC 4.0).

(C) Copyright O.A. Ekeagwu and S.I. Ukpai, 2018 Licensee PAGEPress, Italy

Geriatric Care 2018; 4:7316

doi:10.4081/gc.2018.7316

restricted to not more than 20 hours of work, which provides a standardisation thereby eliminating possible moderating effects of duration of care provided. The impact investigated in the present study is used in terms of effect which includes both burden and benefits in accordance with Fitz-Gibbon's definition. ${ }^{14}$ Measuring the possible benefits is considered necessary to reflect dimensions which can contribute to a positive experience. In addition to the impact, the rationale for working in elderly care was investigated, as it can potentially provide relevant details on approaches towards supporting the caregivers.

\section{Materials and Methods}

Only international students of Anglia Ruskin University who worked in elderly social care were recruited for the study using snowball sampling. The selected sample was chosen to represent the migrant population of elderly caregivers.

\section{Ethical consideration}

This study was conducted according to the guidelines laid down in the Declaration of Helsinki of 1975 and all procedures involving human subjects were approved by the Faculty of Medical Sciences Research Ethics Panel, Anglia Ruskin University (ARU FMS FREP NUMBER 16/17039). Written informed consent was obtained from all subjects.

\section{Data collection}

Data was collected using online surveys. The survey design included selected variables based on their relevance to the objec- 
tives of the research. Questions 1-2 measured the universal variables of age and gender while questions 3-5 sought to enforce the inclusion and exclusion criteria- meaning that for responses to be valid, respondents had to be international students in ARU currently working or have worked as healthcare assistants in elderly care. Questions 6-7 were meant to identify respondents' prior experience working as a caregiver. Questions 8-10 assessed the rationale for the job of choice, while the questions 11-25 measured the impacts of being a formal carer. These included a combination of social, psychological and cultural effects, and possible benefits. Most of the questions, particularly regarding carer burden, were adapted from the Caregiver questionnaires developed by Gater et al. ${ }^{15}$ and Zarit, Reever and BachPeterson. ${ }^{16}$

\section{Pilot survey}

The survey was piloted among 10 university students to ensure the questions were easy to understand, and that the online platform (SurveyMonkey) was efficient. The pilot was undertaken within a day during which the students enrolled via emails, and based on their responses and comments, amendments were made to the survey. Peer assessment for internal validity was also done by administering the questionnaire to 10 students from different faculties, and completed surveys were evaluated for consistency of the responses. Construct validity was also carried out through a review panel of Public Health experts in the University who confirmed the suitability of the survey before use.

\section{Participant recruitment and data collection process}

Given that the study is of prevalence type, precision sample size calculation approach was used. Using this method, an expected prevalence of $5 \%$ was as estimated based on the responses obtained from the 10 students who participated in the pilot study. Also, as recommended by research, ${ }^{17}$ a precision of 0.10 was used at $95 \%$ confidence level, which resulted in 18 , as the minimum number of required subjects for recruitment.

An advert on the university's notice board and university social media page inviting participants-international students working in care to contact the researcher for a survey. Emails were also sent out through the international students' association to members. Once the first carer was identified, they sent links to other carers to participate. The link sent contained a participant information sheet detailing information about the survey

Table 1. Respondents' characteristics, prior care experience and motivation for working in elderly care.

\begin{tabular}{lcc} 
Respondents' characteristics & Number & Proportion (\%) \\
Gender & & 54.5 \\
Male & 18 & 45.5 \\
Female & 15 & \\
Age & & 30.3 \\
18-23 & 10 & 54.5 \\
$24-30$ & 18 & 15.2 \\
31 and older & 5 & \\
Are you an international student? & & 100 \\
Yes & 33 & - \\
No & - & 100 \\
Working or worked in elderly care & & - \\
Yes & 33 & \\
No & - & 54.5 \\
\hline Have you had prior experience in care? & & 45.5 \\
Yes & 18 & \\
No & 15 & 51.5 \\
Area of expertise in prior care experience & & 9.1 \\
Elderly residential care & 17 & 6.1 \\
Mental Health residential care & 3 & 33.3 \\
Other & 2 & \\
None & 11 & 51.6 \\
Motivation for working as a carer & & 24.2 \\
Financial (to support my studies) & 17 & 24.2 \\
Career (to launch me into the job market) & 8 & \\
Experience (to gain experience) & 8 & \\
& & \\
\hline
\end{tabular}

and a link, which prompted them to proceed to the survey on consenting to participate. The survey tool was programmed to prevent multiple responses from a single participant.

Data collection lasted for three weeks after which all data were analysed.

\section{Data analysis}

Collected data was appropriately coded and analysed using the IBM SPSS Statistical 20. Questions 1-4 described demographic variables; questions 5-7 identified areas of elderly care participants were involved with and prior experiences; question 8 sought to define participants' motivations for joining elderly care. All questions were coded appropriately. Questions 9-25 made use of a 5point Likert scale, which ranged from strongly agree to strongly disagree to identify positive and negative impacts of working as a healthcare care assistant. The responses were rated in percentage proportions based on the number of responses.

\section{Results}

Thirty-three respondents were sampled and their characteristics are shown in Table 1. All participants were international students and were either working or had worked in elderly care which was necessary for our study as only that group of students was considered eligible. Nine percent more males than females participated in the study. The majority $(54.5 \%)$ of the respondents were aged 24-30 years, while the least (5\%) number of participants were aged 31 and older.

Also, most participants $(66.7 \%)$ had experience in some form of social care (Table 1).

The motivation for which most $(51.6 \%)$ individuals worked elderly social care jobs was financial, while others were either motivated by their need for working experience or considered it an avenue for launching them into the job market (Table 1).

The assessed impacts of the study include; social, psychological, academic and benefits which are represented in Table 2 . The impact of culture on the perceived burden is also given in Table 2.

In Table 3, factors suspected to be linked with respondents' perceived stress are recorded against the agreement to having perceived stress.

\section{Discussion}

This study sought to investigate the psychological, social, cultural and academic 
impact of elderly care jobs on migrants, as well as identify their rationale for doing the job and determine the possible benefits derived.

\section{Burden of elderly social care}

Research $^{10,11,18,19}$ has affirmed that engaging in care is associated with negative impacts. Results in the present study are in consonance with these studies, revealing that most participants experienced social detachment due to working care jobs (69.7\%), psychological stress $(63.3 \%)$ and reduced study time $(54.6 \%)$. The impact on studies was necessary for our study since the participants were students.

The stress experienced by the carers in our study appears unrelated to their cultural background as majority claimed their cultural background did not make care work difficult. However, we found that most of those who attested to care work being stressful agreed that their cultural background made care work stressful. This was an unexpected discrepancy in the findings and perhaps was the case as none of the migrants would accept that they were struggling with challenges related to cultural adaptation despite that being the case.

Training and prior experience which would generally be perceived to ease jobrelated stress did not appear to ameliorate the perceived stress among the participants.
Rather, most of them who agreed that caring was burdensome had prior experience, and most even felt frustrated because their skills were not adequately utilised. This frustration may be related to the restrictions by protocols and regulations of the nursing homes and care agencies despite the needs of their clients. The UK care system is known to operate by strict adherence to policy such that care actors must comply with their roles in the system, ${ }^{20,21}$ hence unless certified to perform a role, one is forbidden. Although the Care Quality Commission clarifies that such regulations are necessary to protect residents from abuse and related problems, ${ }^{22}$ one may argue that it could reduce the flexibility of care practices and quality of care service delivery eventually. More so, the frustration expressed by the respondents seems to imply their readiness to employ the skills gained despite the stress experienced, which could mean well for the care sector given the encouraging level of motivation.

This study found no such differential impact as more males agreed to care work being stressful converse to extant literature which claims that being female was a determining factor for caregiver burden. ${ }^{8,23,24}$ Previous findings were perhaps related to the historical view of more females being involved in care, ${ }^{25}$ which apparently has changed in present times owing to the growing demand for carers.

\section{Duration of care and development of care and development of a steady relationship}

Carmichael and Charles ${ }^{26}$ claim that working more than 20 hours a week over extended periods of time may increase the perceived burden. The present study could not investigate whether working only 20 hours a week offered a protective effect against stress compared to working longer hours since all participants were restricted to a limit (only 20 hours a week of work). On the other hand, Chiatti and colleagues ${ }^{7}$ observed that with more frequent contact with clients, caregiver burden decreased over time due to development of steady relationships between the carer and the clients. This study could neither confirm nor refute the claim as most respondents were employed via agencies that dispatched them to various care homes at different periods, limiting the propensity to develop any such relationships with clients.

\section{Motivation}

Based on our findings, carers felt motivated by the pecuniary gains of the job in agreement with previous studies.1,24,27 Perhaps this is related to the higher pay rates offered by most care agencies as compared to other jobs considering that the respondents had a 20 -hour per week work restriction. The role of financial gains in motivation for car-

Table 2. Impact of elderly social care on migrant caregivers.

Yes
No
(Agree and
(Disagree or $\quad$ (Neither agree
strongly agree) (\%) strongly disagree) (\%) $\quad$ nor disagree) (\%)

Perceived social impact

Working as a carer means I spend less time with my friends

I have given up a hobby to work as a carer

69.7

48.5

Perceived psychological impact

Caring for others in this role is very stressful

My skills are not fully utilised in this role

I am always treated in the same way as a UK member of staff

I have had enough training and support on how to manage clients in my current role

Perceived academic impact

Working as a carer has made my grades lower

Working as a carer takes time from my studies

Working as a carer has cost me research opportunities

Working as a carer is good for my resume

Cultural impact of perceived burden

My cultural background makes working as a carer difficult

Benefits

Working as a carer helps me save money

Working as a carer helps me understand the UK culture

I have been treated fairly by my employers

Working as a carer aligns with my university course

\begin{tabular}{ccc}
63.3 & 9.4 & 27.3 \\
57.6 & 18.2 & 24.2 \\
75.8 & 3 & 21.2 \\
91 & 3 & 6 \\
\hline
\end{tabular}

\begin{tabular}{lll}
54.6 & 30.2 & 15.2 \\
42.4 & 24.3 & 33.3 \\
18.1 & 36.4 & 45.5 \\
48.5 & 18.2 & 33.3 \\
& & \\
27.3 & 51.5 & 21.2 \\
\hline
\end{tabular}

$\begin{array}{lll}69.7 & 6 & 24.3\end{array}$

$\begin{array}{lll}63.6 & 6.1 & 30.3\end{array}$

$\begin{array}{lll}3 & 75.8 & 21.2\end{array}$

$45.4 \quad 36.4$ 
ers has been argued on finding that more care is done when carers volunteer. ${ }^{28}$ Perhaps the disparate result in our study is peculiar to the demographic characteristics.

\section{Benefits of elderly social care}

One of the criticisms of most of the other instruments used in past research to assess caregiver burden was that they only measured the negative impacts, ${ }^{29}$ leaving unanswered the question of benefits accruing to carers. Our data indicated benefits such as; being good for career prospects due to the training received and providing carers with the opportunity of understanding the UK culture. Perhaps it was for this reason that despite that most perceived the job as stressful, they remained happy to recommend the role to others.

It is noteworthy that despite these benefits, respondents claimed to be treated unfairly by their care employers. Although the exact nature of unfair treatment was not determined in our study, previous research identified incidents such as verbal and physical abuse. ${ }^{3}$ More research is needed in this area especially pertaining to the United Kingdom as it can highlight possible predictors of psychological stress.

\section{Limitations of the study}

Some factors constitute limitations in our study. First, a general approach was used whilst investigating the impact of elderly social care on formal carers, however, this may vary with the type of elderly social care especially as the nursing home attributes and conditions of care may influence the level of burden felt. Also, the results obtained may not be generalizable to carer groups working more than 20 hours a week. This is an important weakness of the study but was unavoidable due to the work limits applicable to the group studied. More so, having used a quantitative design, in-depth perspective of the impact could not be gained. The use of a qualitative design may be necessary to reveal other impact forms not highlighted in our study.

\section{Conclusions}

The delivery of elderly social care without the involvement of migrants may be unsustainable for the provision of continued good outcomes for those in need of care. However, the inherent caregiver burden in elderly social care should be recognised and addressed by all parties involved; the carers, employers, and policymakers. Albeit the avoidance of caregiver burden entirely may be impossible, there should be room for decreasing this impact and providing respite

Table 3. Factors suspected to be linked with respondents' perceived stress.

\begin{tabular}{lc} 
& $\begin{array}{c}\text { Caring for other } \\
\text { is stressful (N) }\end{array}$ \\
Prior experience in care & 17 \\
Yes & 4 \\
No & \\
I have had enough training and support on how to manage clients in my current role & 19 \\
Agree/Strongly agree & 2 \\
Disagree/strongly disagree & \\
\hline My cultural background makes working as a carer difficult & 13 \\
Agree/Strongly agree & 8 \\
Disagree/Strongly disagree & 15 \\
Working as a carer helps me save money & 6 \\
Agree/strongly agree & \\
Disagree/Strongly disagree & 14 \\
\hline Gender & 7 \\
Male & \\
Female & 18 \\
Would you recommend elderly care to others? & 3 \\
Yes & \\
No/Will not say & 20 \\
\hline My skills are not fully utilised in this role & 1 \\
Agree/strongly agree & \\
Disagree/Strongly disagree & \\
\hline
\end{tabular}

for those already affected. Such respite may include counseling, peer support and/or mandatory days off for formal carers after completion of stipulated hours or shifts, and opportunity for newly recruited carers to carry out burden level checks to improve care outcomes.

\section{References}

1. Van-Hooren FJ. Varieties of migrant care work: Comparing patterns of migrant labour in social care. J Eur Soc Policy 2012;22:133-47.

2. Workers Registration Scheme UK. Tier 2 visa restrictions crippling UK care sector study warns. [updated 2015 Sept 20; cited 2017 Feb 17]. Available from: http: //www.workpermit.com/news/2015-1119/tier-2-visa-restrictions-crippling-ukcare-sector-study-warns

3. Clausen T, Hogh A, Carneiro IG, Borg $\mathrm{V}$. Does psychological well-being mediate the association between experiences of acts of offensive behaviour and turnover among care workers? A longitudinal analysis. J Adv Nurs 2013;69: 1301.

4. Simonazzi A. Care regimes and national employment models. Cambridge J Econ 2009;33:211-32.

5. Mcgregor J. Joining the BBC (British Bottom Cleaners): Zimbabwean Migrants and the UK Care Industry. J Ethnic Migrat Stud 2007;33:801-24.

6. Cangiano A, Shutes I, Spencer S, Leeson G. Migrant care workers in ageing societies: research findings; 2009. [cited 2017 Feb 17] Available from: https:// www.compas.ox.ac.uk/media/PR-2009 Care_Workers_Ageing_UK_Executive Summary.pdf

7. Chiatti C, Di Rosa M, Melchiorre MG, et al. Migrant care workers as protective factor against caregiver burden: results from a longitudinal analysis of the EUROFAMCARE study in Italy. Aging Mental Health 2013;17:609-14.

8. Garcés J, Carretero S, Ródenas F, Sanjosé, V. Variables related to the informal caregivers' burden of dependent senior citizens in Spain. Archiv Gerontol Geriatr 2009;48:372-9.

9. Clausen T, Tufte P, Borg V. Why are they leaving? Causes of actual turnover in the Danish eldercare services. J Nurs Manage 2014;22:583.

10. Clark MC, Diamond PM. Depression in family caregivers of elders: A theoretical model of caregiver burden, sociotropy, and autonomy. Res Nurs Health 2010;33:20-34. 
11. Ingleton $\mathrm{C}$, Chatwin J, Seymour J, Payne S. The role of health care assistants in supporting district nurses and family carers to deliver palliative care at home: findings from an evaluation project. J Clin Nurs 2011;20:2043-52.

12. International Organisation for Migration, 2015. Who is a migrant? [online] Available from: http://www. iom.int/who-is-a-migrant? Accessed: 19/06/2016.

13. Giacco D, Matanov A, Priebe S. Providing mental healthcare to immigrants: current challenges and new strategies. Curr Opin Psychiatr 2014; 27:282-8.

14. Fitz-Gibbon CT. Monitoring education: indicators, quality and effectiveness. London: Cassell; 1996.

15. Gater AA, Rofail D, Marshall C, et al. Assessing the impact of caring for a person with schizophrenia: development of the schizophrenia caregiver questionnaire. Patient 2015;8:507-20.

16. Zarit SH, Reever KE, Back-Peterson J. Relatives of the impaired elderly: correlates of feelings of burden. Gerontologist 1980;20:649-55.

17. Pourhoseingholi MA, Vahedi M,
Rahimzadeh M. Sample size calculation in medical studies. Gastroenterol Hepatol Bed Bench 2013;6:14-7.

18. Lloyd C, King R, Chenoweth LI. Social Work, stress and burnout: a review. J Mental Health 2002;11:255-65.

19. Milne A, Larkin M. Knowledge generation about care giving in the UK: a critical review of research paradigms. Health Social Care Commun 2015;23:4-13.

20. Eustis N, Kane R, Fischer L. Home care quality and the home care worker: beyond quality assurance as usual. Gerontologist 1993;33:64.

21. Chen HL. Care workers in long-term care for older people: challenges of quantity and quality. Eur J Social Work 2014;17:383-401.

22. Care Quality Commission. Regulations for service providers and managers. [updated 2017 may 29; cited 2017 Feb 17] Available from: http://www.cqc. org.uk/guidance-providers/regulationsenforcement/regulation-13-safeguarding-service-users-abuse-improper

23. Bedard M, Koivuranta A, Stuckey A. Health impact on caregivers of providing informal care to a cognitively impaired older adult: rural versus urban settings.
Canad J Rural Med 2004;9:15-23. 24. García-Alberca JM, Lara JP, Berthier ML. Anxiety and depression in caregivers are associated with patient and caregiver characteristics in Alzheimer's disease. Int J Psychiatr Med 2011;41: 57-69.

25. Aronson J, Neysmith SM. 'You're not just in there to do the work' Depersonalizing policies and the exploitation of home care workers' labour. Gender Soc 1996;10:59-77.

26. Carmichael F, Charles S. The labour market costs of community care. J Health Econ 1998;17:747-65.

27. Ayalon L, Halevy-Levin S, Ben-Yizhak Z, Friedman G. Family caregiving at the intersection of private care by migrant home care workers and public care by nursing staff. Int Psychogeriatr 2013;25:1463-73.

28. England P. Emerging theories of care work. Annu Rev Soc 2005;1:381-99.

29. Whittingham K, Barnes S, Gardiner C. Tools to measure quality of life and carer burden in informal carers of heart failure patients: a narrative review. Palliat Med 2013;27:596-607. 